\title{
Stochastic thermodynamics for active matter
}

\author{
Thomas Speck \\ Institut für Physik, Johannes Gutenberg-Universität Mainz, Staudingerweg 7-9, 55128 Mainz, Germany \\ PACS 05.70.-a - Thermodynamics \\ PACS 64.75.Xc - Phase separation and segregation in colloidal systems
}

\begin{abstract}
The theoretical understanding of active matter, which is driven out of equilibrium by directed motion, is still fragmental and model oriented. Stochastic thermodynamics, on the other hand, is a comprehensive theoretical framework for driven systems that allows to define fluctuating work and heat. We apply these definitions to active matter, assuming that dissipation can be modelled by effective non-conservative forces. We show that, through the work, conjugate extensive and intensive observables can be defined even in non-equilibrium steady states lacking a free energy. As an illustration, we derive the expressions for the pressure and interfacial tension of active Brownian particles. The latter becomes negative despite the observed stable phase separation. We discuss this apparent contradiction, highlighting the role of fluctuations, and we offer a tentative explanation.
\end{abstract}

Introduction. - Active matter is a somewhat vague term applied to systems driven out of thermal equilibrium by converting stored or locally supplied free energy into directed motion 1]. This notion encompasses systems as disparate as bacterial suspensions 2 and microtubules connected by motor proteins [3]. Of particular interest is the phoretic motion of colloidal Janus particles [4 7 . The question whether active matter could be described in terms of equilibrium thermodynamical concepts 8 has recently attracted considerable interest, in particular the definition of a pressure $9 \sqrt{12}$. While the conventional approach to many non-equilibrium problems is to explicitly formulate and (approximately) solve detailed equations of motion, the power of a thermodynamic description is that it is independent of many of those details and predicts universal bounds.

Over the last two decades, stochastic thermodynamics has evolved into a comprehensive theoretical framework to treat driven systems in contact with a heat reservoir 13 . It applies chiefly to systems with a clear separation of degrees of freedom, where entropy production is due to the slow and observable degrees of freedom following (effective) Markovian stochastic dynamics. So far it has been applied mainly to study colloidal systems and biological matter 14, 15]. The central tenet of stochastic thermodynamics is the first law, not only for mean values but on the level of single trajectories [16]. Fluctuations of work and heat are constrained by fluctuation theorems $17-19$, which ultimately are a consequence of time-reversal sym- metry.

In this paper, we aim to describe particles (cells, bacteria, etc.) that are moving in an aqueous environment. The particles constitute the system specified by their positions (and possibly internal degrees of freedom), while the surrounding solvent takes the role of the reservoir with well-defined temperature $T$, see Fig. 1. The physical picture is that the reservoir is sufficiently large so that the fluctuations remain those of an equilibrium solvent. Moreover, we assume that both the particles and the solvent are enclosed within a container that can take up arbitrary amounts of momentum without being set in motion. This assumption certainly holds for colloidal particles and bacteria moving in a chamber mounted in the laboratory, and indeed for most composite systems attached to the earth (e.g., bacteria in a pond).

Previous discussions on a possible relation to thermodynamics have focused on an effective free energy for active particles 8,20 and fluctuation relations [21]. Here we follow a different strategy that is based on the (virtual) work required to deform a volume of active matter. The first step is to introduce conjugate observables based on the work instead of a free energy. We introduce the concept of effective non-conservative forces for active matter in order to model dissipation. Finally, we investigate in more detail an inhomogeneous system due to phase-separation into a dense and dilute phase [6]. Recently, for this situation it has been found that the interfacial tension (the work to create the interface) is negative 22 but phase 


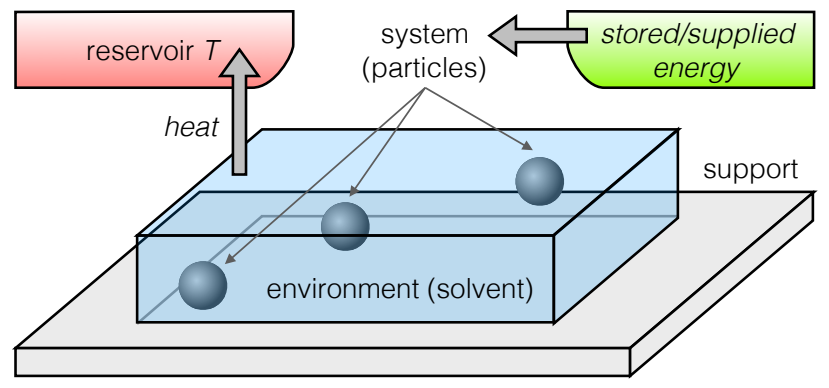

Fig. 1: Typical experimental situation. A microfluidic device or a cell containing a suspension is mounted on a massive support. We identify the suspended "particles" (bacteria, colloidal particles, etc.) as the system and the solvent (plus the surrounding laboratory) as the environment with temperature $T$. The system is externally supplied with energy, which is eventually dissipated as heat.

separation is stable, which can be rationalized within the theoretical framework developed here.

Stochastic thermodynamics. - Let $\boldsymbol{\omega}$ be the configuration of the system with potential energy $U(\boldsymbol{\omega}, \mathbf{X})$, which also depends on a number of external parameters $\mathbf{X}=\left(X_{1}, \ldots\right)$ such as volume $V$. These can be controlled externally while the configuration evolves stochastically due to the coupling with the solvent. Here we assume a time-scale separation such that momenta (and possibly fast degrees of freedom) relax very quickly and thermalize on time scales much shorter than experimentally accessible. The effective Hamiltonian after integrating out the equilibrated degrees of freedom reads

$$
H(\boldsymbol{\omega}, \mathbf{X})=F_{\text {id }}(T, \mathbf{X})+U(\boldsymbol{\omega}, \mathbf{X}),
$$

where $F_{\text {id }}(T, \mathbf{X})$ is the ideal free energy for non-interacting particles in the absence of a potential energy.

The work

$$
\delta \hat{w}=\frac{\partial H}{\partial \mathbf{X}} \cdot \mathrm{d} \mathbf{X}+\mathbf{f} \cdot \mathrm{d} \boldsymbol{\omega}
$$

is composed of two terms: the first term takes into account the work that is spent due to a change of external parameters whereas the second term arises due to nonconservative forces $\mathbf{f}(\boldsymbol{\omega}, \mathbf{X})$. The hat emphasizes that this expression depends on the microstate, while the $\delta$ emphasizes that both work and heat are not exact differentials. The heat that is dissipated then follows from the first law of thermodynamics $\delta \hat{q}=\delta \hat{w}-\mathrm{d} H$ expressing the conservation of energy. The sign is convention, here heat that is dissipated into the solvent is positive. We stress that this balance equation only involves energies and as such is independent of dynamics, in particular hydrodynamic interactions do not enter. Only when taking the average does the dynamics of the system enter.

However, before it is instructive to recall conventional thermodynamics. The systems we are interested in are typically described by a fixed number of constituents in a given volume $V$, the relevant ensemble for which is the canonical ensemble with free energy $F(T, \mathbf{X})=\langle H\rangle-T S$, where $S$ is the entropy and the brackets $\langle\cdot\rangle$ denote the average. The reversible work is given by the differential

$$
\delta w=\mathrm{d} F=\frac{\partial F}{\partial \mathbf{X}} \cdot \mathrm{d} \mathbf{X}=\mathrm{d}\langle H\rangle-T \mathrm{~d} S .
$$

This expression corroborates our identification of stochastic work and heat since $-T \mathrm{~d} S$ is the conventional heat. The work can also be expressed as $\delta w=-\mathbf{K} \cdot \mathrm{d} \mathbf{X}$ with conjugate generalized forces $K_{i}=-\partial F / \partial X_{i}$. In the following we will focus on the extension of two particular conjugate quantities. First, the pressure $p$ conjugate to the volume,

$$
\delta w=\mathrm{d} F=-p \mathrm{~d} V,
$$

and the interfacial tension $\gamma$ describing the reversible work

$$
\delta w=\mathrm{d} F=\gamma \mathrm{d} A
$$

necessary to change the interface area $A$ in an inhomogeneous system at fixed volume $V$. The signs agree with our physical intuition, the pressure opposes the further compression of a system while work has to be spent to extend an interface.

Virtual box change and conjugate stress. We first consider point particles with microstate $\boldsymbol{\omega} \equiv\left\{\mathbf{r}_{k}\right\}$ specified by the positions of all $N$ particles. We aim to calculate the work required by moving all particles according to $\mathbf{r}_{k} \rightarrow \mathbf{r}_{k}^{\prime}=\mathbf{h r}_{k}$, which corresponds to a deformation of the volume $V$ (the "box"). The entries $h_{i j}$ of the matrix $\mathbf{h}$ are now part of the external parameters $\mathbf{X}$. We first consider non-conservative forces to be absent so that the stationary state corresponds to thermal equilibrium. The strain due to this transformation is $\boldsymbol{\epsilon}=\frac{1}{2}\left(\mathbf{h}^{T} \mathbf{h}-\mathbf{1}\right)$ with volume change $V \rightarrow V^{\prime}=V(1+\operatorname{tr} \boldsymbol{\epsilon})$ for small strain 23]. From Eq. (2) the work thus becomes

$$
\delta \hat{w}=V \sum_{i j} \hat{\sigma}_{i j} \mathrm{~d} h_{i j}
$$

with volume $V$ before the transformation and stress tensor

$$
\hat{\sigma}_{i j}(\boldsymbol{\omega})=\left.\frac{1}{V} \frac{\partial H}{\partial h_{i j}}\right|_{\mathbf{h}=\mathbf{1}} .
$$

Eq. (6) is our first central result relating the stress to the work instead of the free energy. Taking the average and exploiting that $\boldsymbol{\sigma}=\langle\hat{\boldsymbol{\sigma}}\rangle$ is a symmetric tensor, one recovers the more conventional expression $\langle\delta \hat{w}\rangle=V \operatorname{tr}(\boldsymbol{\sigma} \mathrm{d} \boldsymbol{\epsilon})$ for the work. Note that the average is with respect to the stationary state before the shape change. Hence, $\delta \hat{w}$ corresponds to the infinitesimal work for a virtual box change without actually having to perform the deformation.

The ideal free energy contributes $-N k_{\mathrm{B}} T \delta_{i j}$ to the stress. The second contribution due to the potential energy reads

$$
\left.\frac{1}{V} \frac{\partial U}{\partial h_{i j}}\right|_{\mathbf{h}=\mathbf{1}}=\frac{1}{V} \sum_{k=1}^{N}\left(\frac{\partial U}{\partial \mathbf{r}_{k}}\right)_{i}\left(\mathbf{r}_{k}\right)_{j}
$$


employing the chain rule, where the subscripts $i$ and $j$ label the vector component. For example, consider a uniform change of the box size with $\mathbf{h}=\lambda \mathbf{1}$. Since $\mathrm{d} \lambda=\mathrm{d} V /(V d)$ we obtain the work $\delta \hat{w}=-\hat{p} \mathrm{~d} V$ with

$$
\frac{1}{d} \operatorname{tr} \hat{\sigma}=-\frac{N k_{\mathrm{B}} T}{V}+\frac{1}{V d} \sum_{k=1}^{N} \frac{\partial U}{\partial \mathbf{r}_{k}} \cdot \mathbf{r}_{k}=-\hat{p}(\boldsymbol{\omega})
$$

which is the microscopic virial expression for the pressure as expected 24]. Here, $V$ is the controlled extensive quantity and the pressure $p=\langle\hat{p}\rangle$ is the conjugate intensive quantity.

Non-conservative forces. We now consider an infinitesimal transformation of the box for systems driven into a non-equilibrium steady state due to nonconservative forces. The total work $\delta \hat{w}=\delta \hat{w}_{\mathrm{ex}}+\delta \hat{w}_{\mathrm{hk}}$ from Eq. (2) can then be split into the "excess" work $\delta \hat{w}_{\text {ex }}$ due to the (explicit or virtual) change of the box (with the relative particle positions fixed) and the "housekeeping" work

$$
\delta \hat{w}_{\mathrm{hk}}=\sum_{k=1}^{N} \mathbf{f}_{k} \cdot \mathrm{d} \mathbf{r}_{k}
$$

due to the evolution of the positions (at fixed box shape). The work due to a box change now reads

$$
\delta \hat{w}_{\mathrm{ex}}=\sum_{i j}\left[\left.\frac{\partial H}{\partial h_{i j}}\right|_{\mathbf{h}=\mathbf{1}}+\sum_{k=1}^{N}\left(\mathbf{f}_{k}\right)_{i}\left(\mathbf{r}_{k}\right)_{j}\right] \mathrm{d} h_{i j} .
$$

Employing Eq. (6) with $\delta \hat{w}_{\mathrm{ex}}$ the microscopic stress tensor thus becomes

$$
\hat{\boldsymbol{\sigma}}(\boldsymbol{\omega})=-\frac{N k_{\mathrm{B}} T}{V} \mathbf{1}+\frac{1}{V} \sum_{k=1}^{N}\left(\mathbf{f}_{k}+\frac{\partial U}{\partial \mathbf{r}_{k}}\right) \mathbf{r}_{k}^{T} .
$$

At this point we have defined a conjugate stress via the work, which reduces to the conventional expression in the absence of non-conservative forces (with $\delta \hat{w}_{\mathrm{hk}}=0$ ).

Again, consider the pressure $p$ in a non-equilibrium steady state now following from $\delta w_{\mathrm{ex}}=-p \mathrm{~d} V$. That this pressure is still an intensive quantity follows from arguments similar to conventional thermodynamics. Suppose we have a system that is partitioned into two volumes $V_{1}$ and $V_{2}$ with total volume $V$. The additive excess work for changing the partition is thus $\delta w_{\text {ex }}=-p_{1} \mathrm{~d} V_{1}-p_{2} \mathrm{~d} V_{2}=$ $-\left(p_{1}-p_{2}\right) \mathrm{d} V_{1}$ due to conservation of the total volume. In a steady state, mechanical stability requires that such a change costs work, $\delta w_{\mathrm{ex}} \geqslant 0$. Hence, $p_{1}=p_{2}$ (since the volume change can take both signs) with $\delta w_{\mathrm{ex}}=0$, which shows that the pressure is uniform in both subsystems.

Inhomogeneous systems. Next, we consider an inhomogeneous system. To this end we generalize the stress tensor Eq. 12 and consider a subregion $R$ with volume $V_{R}$ and sum only over particles $k \in R$ within that subregion. Taking the average $\boldsymbol{\sigma}(\mathbf{r})=\left\langle\hat{\boldsymbol{\sigma}}^{(R)}\right\rangle$ thus leads to a stress tensor that depends on the position of the subregion.
As is usually the case for the transition to a continuous description, in the following we assume that the subregion is sufficiently big to perform the average but small with respect to macroscopic lengths so that $\boldsymbol{\sigma}(\mathbf{r})$ is a differentiable function.

To be more specific, in $d=3$ we consider two regions with different densities that are separated by an interface. We orient the coordinate system so that the interface normal points in $x$-direction. Translational invariance is broken along the normal but still holds perpendicular so that $\boldsymbol{\sigma}(x)$ can only depend on $x$. We consider a virtual transformation

$$
\mathbf{h}=\left(\begin{array}{ccc}
\lambda_{\perp} & 0 & 0 \\
0 & \lambda_{\|} & 0 \\
0 & 0 & \lambda_{\|}
\end{array}\right)
$$

so that the box changes independently in the directions parallel and perpendicular to the interface. The excess work thus becomes

$$
\delta \hat{w}_{\mathrm{ex}}=\sum_{R} V_{R}\left[\hat{\sigma}_{x x}^{(R)} \mathrm{d} \lambda_{\perp}+\left(\hat{\sigma}_{y y}^{(R)}+\hat{\sigma}_{z z}^{(R)}\right) \mathrm{d} \lambda_{\|}\right],
$$

where we have split the total volume $V$ into nonoverlapping stripes. The changes of volume and interfacial area $A$ follow as $\mathrm{d} V_{R}=V_{R} \mathrm{~d} \lambda_{\perp}+2 V_{R} \mathrm{~d} \lambda_{\|}$and $\mathrm{d} A=2 A \mathrm{~d} \lambda_{\|}$, respectively, with $V_{R}=A \Delta x_{R}$ and $\Delta x_{R}$ the width of stripe $R$. For fixed volume $\left(\mathrm{d} V_{R}=0\right)$ we thus obtain $\delta \hat{w}_{\mathrm{ex}}=\hat{\gamma} \mathrm{d} A$, whereby

$$
\hat{\gamma}(\boldsymbol{\omega}) \equiv \sum_{R} \Delta x_{R}\left[-\hat{\sigma}_{x x}^{(R)}+\frac{1}{2}\left(\hat{\sigma}_{y y}^{(R)}+\hat{\sigma}_{z z}^{(R)}\right)\right]
$$

can be identified as an interfacial tension. Performing the average together with the limit $\Delta x_{R} \rightarrow 0$ of infinitesimal thin stripes thus leads to the integral

$$
\gamma=\langle\hat{\gamma}\rangle=\int \mathrm{d} x\left[-\sigma_{\perp}(x)+\sigma_{\|}(x)\right]
$$

with normal stress $\sigma_{\perp}(x)=\left\langle\hat{\sigma}^{(R)}\right\rangle$ and tangential stress $\sigma_{\|}(x)=\left\langle\hat{\sigma}_{y y}^{(R)}\right\rangle=\left\langle\hat{\sigma}_{z z}^{(R)}\right\rangle$. In bulk both stresses are equal and the integral only picks up contributions from the interfacial region. We have thus derived another conjugate quantity, the interfacial tension $\hat{\gamma}(\boldsymbol{\omega})$, which describes the work that would be required to change the interfacial area while holding the total volume constant. Eq. $\sqrt{16}$ is the same as what Kirkwood and Buff have derived using mechanical arguments [25], which thus also holds in nonequilibrium steady states.

Effective swim forces. - We now apply the ideas developed so far to self-propelled particles. Every particle is described by its position $\mathbf{r}_{i}$ and an orientation $\mathbf{e}_{i}$ with unity magnitude, $\left|\mathbf{e}_{i}\right|=1$, so that the full configuration is specified by $\boldsymbol{\omega} \equiv\left\{\mathbf{r}_{i}, \mathbf{e}_{i}\right\}$. We adopt the following perspective: by some mechanism available free energy (either stored or due to a local gradient) is converted into motion; however, we do not resolve the details of this mechanism 
explicitly. Rather, we exploit that due to this motion the particles exert a force $\mathbf{f}_{k}(\boldsymbol{\omega})$ onto the surrounding solvent, the hydrodynamic drag, which in general depends on the details of the generated flow profile. The role of the nonconservative forces $\mathbf{f}_{k}$ is thus to describe the dissipation that is required to maintain the directed motion.

This perspective follows the approach of stochastic thermodynamics: we split the total system into the actual system (the particles) and the environment (the solvent). Particles are supplied with energy from the outside by some means. For bacteria, one could image that some of that supplied "fuel" is used for the internal metabolism, in which case the internal state has to be included in the energy balance. On the other hand, colloidal particles are inert and so all of this energy is dissipated into the solvent. To employ the first law we need to model this dissipation. To this end we ask for the effective force that would be necessary to achieve a certain speed. We stress that this force is an effective force that does not necessarily appear in the equations of motion (but see also Refs. 26, 27).

Active Brownian particles. We now consider a more specific, minimal model for active colloids: active Brownian particles $(\mathrm{ABP})$, which combines volume exclusion with persistence of motion. This model neglects hydrodynamic interactions as well as long-ranged phoretic interactions. The potential energy $U(\boldsymbol{\omega})=\sum_{i<j} u\left(\left|\mathbf{r}_{i}-\mathbf{r}_{j}\right|\right)$ with conservative forces $\mathbf{F}_{k}=-\nabla_{k} U$ stems from pairwise interactions with pair potential $u(r)$, and depends on particle positions only. The equations of motion for the $N$ spherical particles read

$$
\dot{\mathbf{r}}_{k}=v_{0} \mathbf{e}_{k}+\mu_{0} \mathbf{F}_{k}+\boldsymbol{\xi}_{k}
$$

where the noise $\boldsymbol{\xi}_{k}$ models the solvent at temperature $T$ with correlations $\left\langle\xi_{k, i}(t) \xi_{l, j}\left(t^{\prime}\right)\right\rangle=2 \mu_{0} k_{\mathrm{B}} T \delta_{k l} \delta_{i j} \delta\left(t-t^{\prime}\right)$. Every particle is propelled with the same speed $v_{0}$ along its orientation $\mathbf{e}_{i}$, which undergoes free diffusion with diffusion coefficient $D_{\mathrm{r}}$. Since we neglect hydrodynamic interactions, the hydrodynamic forces are simply

$$
\mathbf{f}_{k}=-\frac{v_{0}}{\mu_{0}} \mathbf{e}_{k} \equiv-f_{0} \mathbf{e}_{k}
$$

which is the frictional force due to a sphere moving with constant velocity $v_{0}$.

The average rate for the housekeeping work Eq. 10 becomes

$$
\dot{w}_{\mathrm{hk}}=-f_{0} \sum_{k=1}^{N}\left\langle\mathbf{e}_{k} \cdot \dot{\mathbf{r}}_{k}\right\rangle=-N f_{0} v .
$$

To calculate the effective speed $v \equiv\left\langle\mathbf{e}_{k} \cdot \dot{\mathbf{r}}_{k}\right\rangle$ we insert Eq. 17). The term $\left\langle\mathbf{e}_{k} \cdot \boldsymbol{\xi}_{k}\right\rangle=0$ vanishes since orientations and translational noises are uncorrelated. The other two terms lead to $v=v_{0}-\mu_{0} \bar{\rho} \zeta$ with force imbalance coefficient $\zeta$, see Ref. 28 for details. The effective speed $v<v_{0}$ is reduced compared to the free propulsion speed $v_{0}$ due to interactions with other particles blocking the directed motion. However, on average particles still move in the direction of the propulsion and hence $v>0$, which implies that $\dot{w}_{\mathrm{hk}}<0$ is negative. This exemplifies our argument that the particles spent work on the solvent through the hydrodynamic drag.

Pressure. We now calculate the microscopic expression for the pressure of ABPs by inserting the effective forces Eq. 18) into the stress Eq. 12. For a uniform box change we thus obtain

$$
\hat{p}(\boldsymbol{\omega})=\bar{\rho} k_{\mathrm{B}} T+\hat{p}^{(\mathrm{i})}(\boldsymbol{\omega})+\hat{p}^{(\mathrm{a})}(\boldsymbol{\omega}),
$$

which can be written as sum of three contributions. The first term is the ideal gas pressure with global density $\bar{\rho} \equiv$ $N / V$. The second term

$$
\hat{p}^{(\mathrm{i})}(\boldsymbol{\omega})=\frac{1}{2 A} \sum_{k<l}^{N} w\left(\left|\mathbf{r}_{k}-\mathbf{r}_{l}\right|\right)
$$

with $w(r) \equiv-r u^{\prime}(r)$ takes into account the direct interactions between particles through the pair potential $u(r)$. It reduces to an expression that manifestly involves only particle distances. The third term is the contribution

$$
\hat{p}^{(\mathrm{a})}(\boldsymbol{\omega})=\frac{1}{2 A} \frac{v_{0}}{\mu_{0}} \sum_{k=1}^{N} \mathbf{e}_{k} \cdot \mathbf{r}_{k}
$$

of the non-conservative forces Eq. (18) due to the selfpropulsion. Note that here the absolute particle positions enter. Our approach via the excess work for a virtual deformation thus leads to the same expression Eq. 20 for the pressure derived previously following quite different arguments 912 .

Interfacial tension. - For sufficiently high densities and propulsion speeds, suspensions of ABPs interacting via a repulsive pair potential separate into a dilute and a dense phase, a phenomenon that strongly resembles passive liquid-gas phase separation but has a purely dynamical origin 28 31]. In Ref. 22 it has been found that $\gamma<0$ becomes negative, which implies that extending the interface releases work. In a passive system this would lead to a proliferation of interfaces and finally to homogenization, quite in contrast to the observed stable phase separation of ABPs. This result is instructive because it highlights two issues: (i) not all properties that hold in equilibrium are transferable to driven systems and (ii) the role of fluctuations. In equilibrium, the latter are determined by the free energy, the same quantity that determines the pressure, cf. Eq. (4). This connection no longer holds away from equilibrium.

Constructing the interface. Following Ref. 22, we consider a two-dimensional, large but finite system with a single interface that spans the full system. This interface is not static but changes constantly due to fluctuations. A convenient concept is the Gibbs dividing surface constructed by extended the bulk densities $\rho_{ \pm}$such that the 
(a)

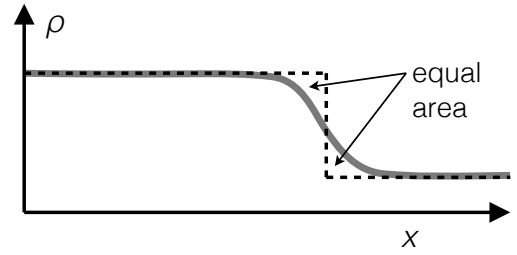

(b)

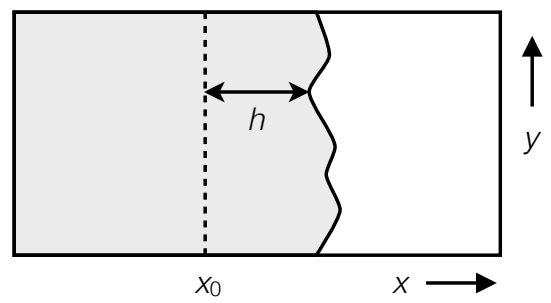

Fig. 2: Gibbs dividing line in two dimensions. (a) Average density profile (solid line) as a function of $x$. The position of the step profile (dashed line) is determined by an equal area rule. (b) Instantaneous interface $x_{0}+h(y)$.

position of the dividing line is determined by the conservation of density, see Fig. 2(a). Without loss of generality, we assume that the normal of the (averaged) interface coincides with the $x$-axis. We further assume that we can construct the Gibbs dividing line for thin vertical stripes in order to obtain an instantaneous interface $x=x_{0}+h(y)$ with respect to a reference position $x_{0}$, see Fig. 2(b). Note that we ignore overhangs. Following capillary wave theory, in a finite system with dimensions $L_{x} \times L_{y}$ and periodic boundaries, we decompose the interfacial profile into complex Fourier modes

$$
h(y)=\sum_{q} h_{q} e^{\mathrm{i} q y}, \quad h_{q}=\frac{1}{L_{y}} \int_{0}^{L_{y}} \mathrm{~d} y h(y) e^{-\mathrm{i} q y}
$$

with $h_{-q}=h_{q}^{*}$, where $h_{q}^{*}$ denotes the conjugate complex. The instantaneous length of the interface can then be calculated as

$$
\hat{\ell}=\int_{0}^{L_{y}} \mathrm{~d} y \sqrt{1+\left[\partial_{y} h\right]^{2}} \approx L_{y}+\frac{1}{2} L_{y} \sum_{q} q^{2}\left|h_{q}\right|^{2},
$$

where we have approximated $\sqrt{1+x} \approx 1+x / 2$.

Interfacial width. In thermal equilibrium, the probability to observe an interface with length $\ell$ is given by the change of free energy (viz., the reversible work to deform the flat interface),

$$
p(\ell) \propto e^{-\left[F(\ell)-F\left(L_{y}\right)\right] / k_{\mathrm{B}} T} \propto e^{-\gamma_{\mathrm{eq}} \ell / k_{\mathrm{B}} T} .
$$

For the second result we have expanded the free energy to linear order and used $\gamma_{\mathrm{eq}}=\left.\partial_{\ell} F\right|_{L_{y}}$, cf. Eq. (5). Following the usual argument, higher orders become negligible in the limit of large system size since $\partial_{\ell}^{2} F \sim \mathcal{O}\left(L_{y}^{-1}\right)$.

In agreement with the observed stable phase separation, for ABPs we assume a distribution $p(\ell)$ of interfacial lengths to exist. Following the same argument as above, we can thus approximate

$$
p(\ell) \asymp e^{-\Omega(\ell)} \propto e^{-\kappa \ell}
$$

to leading order with unknown interfacial stiffness $\kappa=$ $\left.\partial_{\ell} \Omega\right|_{L_{y}}>0$. Our use of the term "stiffness" is based on its role in capillary wave theory for interface fluctuation in equilibrium, where $\kappa_{\mathrm{eq}}=\gamma_{\mathrm{eq}} / k_{\mathrm{B}} T$ holds.

With this approximation we can determine the probability of the Fourier coefficients $h_{q}$ in the non-equilibrium steady state. Employing Eq. 24, to lowest order the modes are independent and Gaussian,

$$
P\left(\left\{h_{q}\right\}\right) \propto e^{-\kappa \hat{\ell}} \propto \exp \left\{-\frac{1}{2} \kappa L_{y} \sum_{q} q^{2}\left|h_{q}\right|^{2}\right\},
$$

so that we can immediately read off the fluctuations $\left\langle\left|h_{q}\right|^{2}\right\rangle=\left(\kappa L_{y} q^{2}\right)^{-1}$. With this result we can finally calculate the average width of the interface as

$$
w^{2}=\frac{1}{L_{y}} \int_{0}^{L_{y}} \mathrm{~d} y\left\langle[h(y)]^{2}\right\rangle=\sum_{q}\left\langle\left|h_{q}\right|^{2}\right\rangle .
$$

To leading order, the total width is then given by an unknown "intrinsic" width $w_{0}$ and the contributions due to the capillary waves with $|q|>0$ and $q=\left(2 \pi / L_{y}\right) n$,

$$
w^{2}=w_{0}^{2}+\frac{2}{\kappa L_{y}} \sum_{q>0} \frac{1}{q^{2}}=w_{0}^{2}+\frac{L_{y}}{12 \kappa},
$$

for which the linear dependence on $L_{y}$ has been confirmed numerically [22].

Discussion. To relate tension and stiffness, we start by rearranging their relation in equilibrium,

$$
\gamma_{\mathrm{eq}}=\frac{w}{\Delta \ell}=\kappa_{\mathrm{eq}} k_{\mathrm{B}} T
$$

where $w$ is the (reversible) work required to extend the interface by $\Delta \ell$. Note that this equation has the form of a fluctuation-dissipation relation, where the tension (dissipation) is related to the stiffness (fluctuations) through the thermal energy $k_{\mathrm{B}} T$. The physical picture is that, for a fluctuation away from the flat profile, the system "borrows" energy from the heat reservoir through a collective fluctuation.

The conjecture of Ref. 22 has been that a relation analogous to Eq. (30) also holds for ABPs,

$$
\gamma=\frac{w_{\mathrm{ex}}}{\Delta \ell}=\kappa \frac{\dot{w}_{\mathrm{hk}} \tau}{N}=\kappa\left(-f_{0} \ell_{\mathrm{p}}\right),
$$

where the thermal energy is now replaced by the housekeeping work per particle (during the time $\tau$ required to extend the interface by $\Delta \ell$ ), which is given by the hydrodynamic friction force $f_{0}$ times the distance $\ell_{\mathrm{p}}(\tau)=v \tau$. The physical picture is that the housekeeping work sets the scale for the energy that is available and thus determines 
(a)

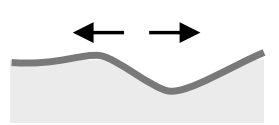

(b)

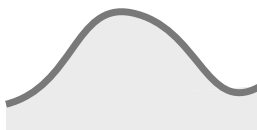

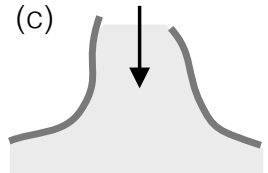

Fig. 3: Fluctuations of the interface. (a) Forces within the interface are extensile (arrows), for which fluctuations lead to (b) buckling and finally (c) rupture of the interface with particles entering (arrow).

the fluctuations. Since $\kappa>0$, Eq. (31) indeed predicts a negative tension $\gamma<0$. In Ref. 22 it has been shown that using $\ell_{\mathrm{p}} \approx v_{0} / D_{\mathrm{r}}$ leads to a quantitative agreement between the stiffness $\kappa$ extracted from measured interfacial widths using Eq. (29) and the tension $\gamma$ calculated via Eq. 16).

Fig. 3 sketches a possible scenario rationalizing a negative tension with a positive stiffness based on the strong fluctuations of the interface, which in simulations is seen to "rupture" and then to reform. Since the tension is negative, the interface is extensile, i.e., the active forces stretch the interface [Fig. 3(a)]. Fluctuations perpendicular to the interface [Fig. 3(b)] are thus amplified and finally the interface "ruptures" [Fig. 33(c)]. Due to the polarization of particles at the interface pointing towards to denser region, this is followed by an incursion of particles stabilizing phase separation. Note that a somewhat related scenario based on defects has been described for active nematic-isotropic interfaces [32] Moreover, simulations in three dimensions, for which fluctuations are even stronger, show clear evidence for these collective incursions from the interface into the dense domains 33 .

Conclusions. - In this letter we have introduced two fundamental concepts: (i) conjugate observables out of equilibrium based on (fluctuating) work, and (ii) the use of effective non-conservative forces to model the dissipated work necessary to sustain the directed motion of active matter. As a first step we have applied these ideas to the minimal model of active Brownian particles. The result for the pressure agrees with previous derivations. For the interfacial tension we have proposed a relation connecting fluctuations with the housekeeping work. While here we have considered large systems neglecting boundaries and walls, these have a profound influence on active matter and will have to be addressed in future work.

\section{$* * *$}

The DFG is acknowledged for financial support within priority program SPP 1726 (grant number SP 1382/3-1).

\section{REFERENCES}

[1] Vicsek T. and Zafeiris A., Phys. Rep., 517 (2012) 71.
[2] Wensink H. H., Dunkel J., Heidenreich S., Drescher K., Goldstein R. E., Löwen H. and Yeomans J. M., Proc. Natl. Acad. Sci. U.S.A., 109 (2012) 14308.

[3] Sanchez T., Chen D. T. N., DeCamp S. J., Heymann M. and Dogic Z., Nature, 491 (2012) 431.

[4] Theurkauff I., Cottin-Bizonne C., Palacci J., Ybert C. and Bocquet L., Phys. Rev. Lett., 108 (2012) 268303.

[5] Palacci J., Sacanna S., Steinberg A. P., Pine D. J. and Chaikin P. M., Science, 339 (2013) 936.

[6] Buttinoni I., Bialké J., Kümmel F., Löwen H., Bechinger C. and Speck T., Phys. Rev. Lett., 110 (2013) 238301.

[7] Bialké J., SpeCK T. and Löwen H., J. Non-Cryst. Solids, 407 (2015) 367.

[8] Takatori S. C. and Brady J. F., Phys. Rev. E, 91 (2015) 032117.

[9] Takatori S. C., Yan W. and Brady J. F., Phys. Rev. Lett., 113 (2014) 028103.

[10] Solon A. P., Stenhammar J., Wittkowski R., Kardar M., Kafri Y., Cates M. E. and Tailleur J., Phys. Rev. Lett., 114 (2015) 198301.

[11] Solon A. P., Fily Y., Baskaran A., Cates M. E., Kafri Y., Kardar M. and Tailleur J., Nature Phys., 11 (2015) 673.

[12] Winkler R. G., Wysocki A. and Gompper G., Soft Matter, 11 (2015) 6680.

[13] Seifert U., Rep. Prog. Phys., 75 (2012) 126001.

[14] Collin D., Ritort F., Jarzynski C., Smith S., Tinoco I. and Bustamante C., Nature, 437 (2005) 231.

[15] Blickle V., Speck T., Helden L., Seifert U. and Bechinger C., Phys. Rev. Lett., 96 (2006) 070603.

[16] Sekimoto K., Prog. Theor. Phys. Supp., 130 (1998) 17.

[17] Kurchan J., J. Phys. A: Math. Gen., 31 (1998) 3719.

[18] Lebowitz J. L. and Spohn H., J. Stat. Phys., 95 (1999) 333.

[19] Seifert U., Phys. Rev. Lett., 95 (2005) 040602.

[20] Cates M. E. and Tailleur J., Annu. Rev. Condens. Matter Phys., 6 (2015) 219.

[21] Ganguly C. and Chaudhuri D., Phys. Rev. E, 88 (2013) 032102.

[22] Bialké J., Siebert J. T., Löwen H. and Speck T., Phys. Rev. Lett., 115 (2015) 098301.

[23] Landau L. and Lifshitz E., Theory of Elasticity 2nd Edition Vol. 6 (Pergamon Press, Oxford) 1970.

[24] Hansen J. and McDonald I., Theory of Simple Liquids 3rd Edition (Academic Press, Amsterdam) 2006.

[25] Kirkwood J. G. and Buff F. P., J. Chem. Phys., 17 (1949) 338.

[26] ten Hagen B., Wittkowski R., Takagi D., Kümmel F., Bechinger C. and Löwen H., J. Phys.: Condens. Matter, 27 (2015) 194110.

[27] Yan W. and Brady J. F., Soft Matt., 11 (2015) 6235.

[28] Speck T., Menzel A. M., Bialké J. and Löwen H., J. Chem. Phys., 142 (2015) 224109.

[29] Fily Y. and Marchetti M. C., Phys. Rev. Lett., 108 (2012) 235702.

[30] Cates M. E. and Tailleur J., EPL, 101 (2013) 20010.

[31] Bialké J., Löwen H. and Speck T., EPL, 103 (2013) 30008.

[32] Blow M. L., Thampi S. P. and Yeomans J. M., Phys. 
Rev. Lett., 113 (2014) 248303.

[33] Wysocki A., Winkler R. G. and Gompper G., EPL, 105 (2014) 48004. 